Artículo Original

\title{
Etograma y análisis de los sonidos realizados por los machos durante la cópula en Colletes musculus (Friese) (Hymenoptera: Colletidae)
}

\author{
Ethogram and analysis of sounds performed by males during the copula in \\ Colletes musculus (Friese) (Hymenoptera: Colletidae)
}

Elizabeth Chiappa ${ }^{1}$, Víctor Mandujano ${ }^{1}$ y Guillermo Riveros ${ }^{1}$

${ }^{1}$ Facultad de Ciencias, Universidad de Playa Ancha. Avda. Leopoldo Carvallo $N^{\circ} 270$, Valparaíso, Chile. 泉echiappa@upla.cl

\section{ZooBank: urn:lsid:zoobank.org:pub: 4A544F1D-CCC4-48ED-8FAE-9FD394D09C16 https: / / doi.org/10.35249/ rche.46.4.20.01}

Resumen. En el presente estudio se describe el comportamiento de cópula de los machos de Colletes musculus (Friese). Se analizan los sonidos producidos por ellos mediante vibración de las alas durante el apareamiento. Reevaluamos la conducta reproductiva en machos de C. musculus, basándonos en el análisis de las interacciones de los comportamientos reproductivos filmados en una población del sur de Chile. Las vibraciones que producen los machos durante la cópula podrían servir para cambiar la receptividad de las hembras. Se describen las conductas registradas antes, durante y después de la cópula las que se representan en un etograma, que resume el comportamiento de cópula de los machos.

Palabras clave: Abeja solitaria, bioacústica, Chile, comportamiento reproductivo.

Abstract. In the present study, male's mating behavior of Colletes musculus (Friese) is described. We analize the sounds produced by them through wings vibratios during copulation. We reevaluated reproductive behavior in males of $C$. musculus, based on the analysis of interactions of reproductive behaviors filmed in a population in southern Chile. The vibrations produced by males during the genital contact may serve to change the receptivity of the female. Behaviors recorded before, during and after copulation are described, which are represented in an ethogram, which summarizes the copulation behavior of males.

Key words: Bioaccoustic, Chile, mating behaviour, solitary bee.

\section{Introducción}

En Chile se han descrito 27 especies del género Colletes Latreille (Montalva y Ruz 2010), sin embargo se ha informado muy poco sobre la biología y conducta de esas especies. Sobre C. musculus (Friese), sólo Janvier (1926) ha realizado una breve descripción de los nidos y hace poco se han descrito los estados inmaduros en detalle (Chiappa et al. 2018).

En insectos, muchos estudios sobre comunicación antes o durante la cópula se refieren principalmente a comunicación química (Alcock y Buchmann 1985; Cade 1985; Vander Meer et al. 1998; Leonhardt et al. 2016). Las feromonas que atraen a las potenciales

Recibido 16 Junio 2020 / Aceptado 24 Septiembre 2020 / Publicado online 30 Octubre 2020

Editor Responsable: José Mondaca E. 
parejas son generalmente emitidas por las hembras y no por los machos (Thornhill 1979; Hölldobler y Wilson 1990).

En abejas, se ha realizado poca investigación sobre el rol de la comunicación a través del sonido durante la cópula y sobre la relevancia de este tipo de interacción, especialmente cuando las especies no poseen órganos especializados para emitir sonidos (Conrad et al. 2010). Algunos trabajos mencionan la comunicación mediante el sonido producido durante la cópula en especies de la superfamilia Apoidea, indicando que son producidos por estructuras que se frotan una contra la otra (estridulación) como es el caso de Centris mixta Friese y C. pallida Fox o como resultado de la vibración producida por los músculos indirectos del vuelo en Colletes cunicularius (Linnaeus), Bembix rostrata (Fabricius) o en Osmia rufa (Linnaeus) (Markl 1983; Alcock y Buchmann 1985; Larsen et al. 1986; Wisclo et al. 1992; Toro y Riveros 1998; Conrad et al. 2010).

En varias especies de abejas, el zumbido que regularmente producen los machos son emitidos durante la cópula y no al término de ella como se ha observado en Centris pallida y C. mixta (Alcock y Buchmann 1985; Toro y Riveros 1998). Se ha sugerido que el rol de las señales acústicas y vibracionales emitidas durante la cópula por los machos de especies de Apoidea podrían ser señales indicativas hacia las hembras sobre la calidad o estado del macho. Estas señales podrían indicar una mayor energía o una mayor viabilidad reproductiva, características importantes para la selección de pareja (Toro y Riveros 1998; Hill 2008).

Respecto al establecimiento de etapas conductuales generadas durante la cópula, no se han realizado estudios en Apoidea chilenos, excepto para la avispa endémica Sphex latreillei (Lepeletier) (Apoidea: Sphecidae: Cabroninae) con referencia particular a la selección sexual realizada por las hembras durante la cópula (Mandujano et al. 2016).

La ventaja de realizar un etograma de la conducta de apareamiento, es que se puede obtener la lista de conductas organizadas en categorías. Se obtiene, además, la descripción de los elementos y la función de cada comportamiento significativo para los individuos, reflejando la estereotipia de las conductas. En el caso de C. musculus ayuda a interpretar el sistema de apareamiento de la abeja.

El objetivo del presente estudio es describir y caracterizar el comportamiento de cópula en $C$. musculus agregando un elemento poco utilizado para el análisis conductual: el sonido que producen los machos durante la cópula, el cual sólo se ha analizado en Chile para Centris mixta (Hymenoptera: Apoidea: Centridini) (Toro y Riveros 1998). Además, como resultado de la descripción se presenta un etograma que resume y representa los diferentes comportamientos que los machos realizan durante la cópula.

\section{Metodología}

El estudio se realizó en una nidificación cerca de la localidad de Puerto Saavedra, específicamente en la área de Boca Budi (3856’S - 73²0’W), en la Región de La Araucanía. Las observaciones se hicieron durante dos épocas reproductivas de la especie, desde octubre a enero en los años 2015 y 2016. El agregado de nidos, de $15 \mathrm{~m}$ de largo x $1 \mathrm{~m}$ de ancho, se encontraba en una terraza marina a 10-12 msnm, bordeando la playa, en un suelo de arena y rodeada de escasa vegetación, compuesta principalmente por plantas de pequeña altura. Los nidos estaban dispuestos muy cercanos entre ellos en un desnivel que terminaba en una planicie (ver Fig. 1 en Chiappa et al. 2018).

Las filmaciones de los especímenes en cópula fueron tomadas del 13 al 15 de noviembre del 2015, entre las 9:00 y las 17:00. La duración total de grabación fue de 53 minutos 44 segundos, de ellos 21 minutos 41 segundos corresponden a 7 cópulas completas, que se analizaron para identificar y establecer unidades de comportamiento (Tabla 1) (Peláez y Veá 1997). Además, se realizaron observaciones cualitativas y cuantitativas sobre la cópula. 
Las cópulas se filmaron en terreno para no afectar la calidad de la grabación, a un metro de distancia con una desviación de $0^{\circ}$ con una cámara Samsung HMX-H300 Full HD Camcorder, que tiene un micrófono estéreo omnidireccional. También los ejemplares en cópula, se fotografiaron en el laboratorio, con el software TS View 7S y cámara digital Tucsen bajo lupa estereoscópica Olimpus SZ61 para medir la distancia intertegular, que refleja el tamaño de las abejas (Cane 1987).

En el caso de los sonidos, utilizamos el software Spectra Plus v.5.01 para analizar 25 minutos de grabaciones sonoras de la cópula de C. musculus y 150 emisiones sonoras producidas por los machos. Para relacionarlo con el comportamiento de cópula, cada emisión de sonido se caracterizó bioacústicamente determinando los valores de los siguientes parámetros acústicos: frecuencia $(\mathrm{Hz})$, duración de las notas (ms), período de tiempo entre la emisión de las notas (segundos) y la presencia de armónicos, con la frecuencia respectiva del armónico fundamental y el armónico que concentra la energía de emisión de las notas.

Con el fin de analizar las grabaciones de video, para evaluar las unidades de comportamiento identificadas $(\mathrm{n}=7)$, se realizó una tabla de contingencia $7 \times 7$. Cada cuadro representa la transición de un comportamiento a otro, considerando que la probabilidad de una unidad de comportamiento dada depende sólo de la identidad de la unidad de comportamiento anterior (cadena de Markov de primer orden) (Fagen y Young 1978).

Posteriormente, se definirieon las transiciones significativas utilizando la fórmula $Y=\left(x_{i j}-m_{i j}\right) /\left(m_{i j}\right)^{1 / 2}$, donde si, $|Y|>\sqrt{x^{2}{ }_{0,05}, g . l} . / R^{2}$, entonces la transición (act $\mathrm{i} \rightarrow$ act $\mathrm{j}$ ) ocurre con una frecuencia que difiere significativamente $(\mathrm{p}<0,05)$ de la ocurrencia aleatoria. Si $Y>0$ y la transición se considera significativa, el acto i lleva directamente al acto j; cuando $\mathrm{Y}<0$ y la transición se considera significativa, el acto i inhibe el acto j (Fagen y Young 1978).

$$
S l i=\sqrt{\left[\frac{\sum(P i j)^{2}+\left(\sum P i j\right)^{2} / r i}{(1+1 / r i)}\right]}
$$

El grado de estereotipia en cada paso de la secuencia de comportamiento se calculó a través del índice de estereotipia para cada transición (Sli). Para obtener el índice de estereotipia de la secuencia de comportamiento global (SI), el valor SIi para cada transición se ponderó por su frecuencia (Sl, parciales). La suma de estos valores dio como resultado el valor del índice de estereotipia para la secuencia global (SI). Finalmente, se construyó un etograma, a partir de las transiciones significativas obtenidas.

\section{Resultados}

En noviembre, se observó una gran actividad de hembras volando en el área y también machos que intentaban copular en la entrada o cerca de la entrada del nido (Chiappa et al. 2018).

La cópula presenta un rango de duración, entre 5 y $12 \min (\mathrm{n}=7)$. La pareja en cópula es acosada con frecuencia por otros machos, que forman pequeños agregados de 3-5 individuos intentando copular, pero que se alejan rápidamente cuando uno de ellos tiene éxito y consigue inmovilizar a la hembra y rechaza a cada rival que pretende reemplazarlo. En todas las observaciones se observó que la hembra permanece inmovilizada durante el apareamiento, de acuerdo a los pasos que se describe a continuación.

El apareamiento de C. musculus consta de siete pasos y seis transiciones, a) patrullaje de los machos, que intentan localizar a una hembra que está en el suelo cerca del área de anidación, b) contacto, el macho se acerca a la hembra volando y se coloca en paralelo sobre el dorso de la hembra, c) montaje, d) el macho agarra las alas de la hembra por la vena costa con sus patas delanteras, segundo y tercer par de patas quedan adosados a los 
lados del abdomen de la hembra e) copula, insertando sus genitales. Es en este momento cuando el macho mueve sus alas generando una vibración, intercalando con movimientos abdominales anteroposteriores, mantiene siempre sus antenas dirigidas hacia arriba, el macho mantiene sus alas plegadas sobre el abdomen, alternando periodicamente el movimiento de las alas con los movimientos abdominales $(n=7)$.

Inmediatamente luego de la cópula, f) desajueste de la genitalia, el macho suele permanecer por aproximadamente 15 segundos cerca de la hembra, mientras esta limpia el abdomen con las patas posteriores por 34 segundos $(n=7)$, luego la pareja se separa y el g) macho vuela fuera del área.

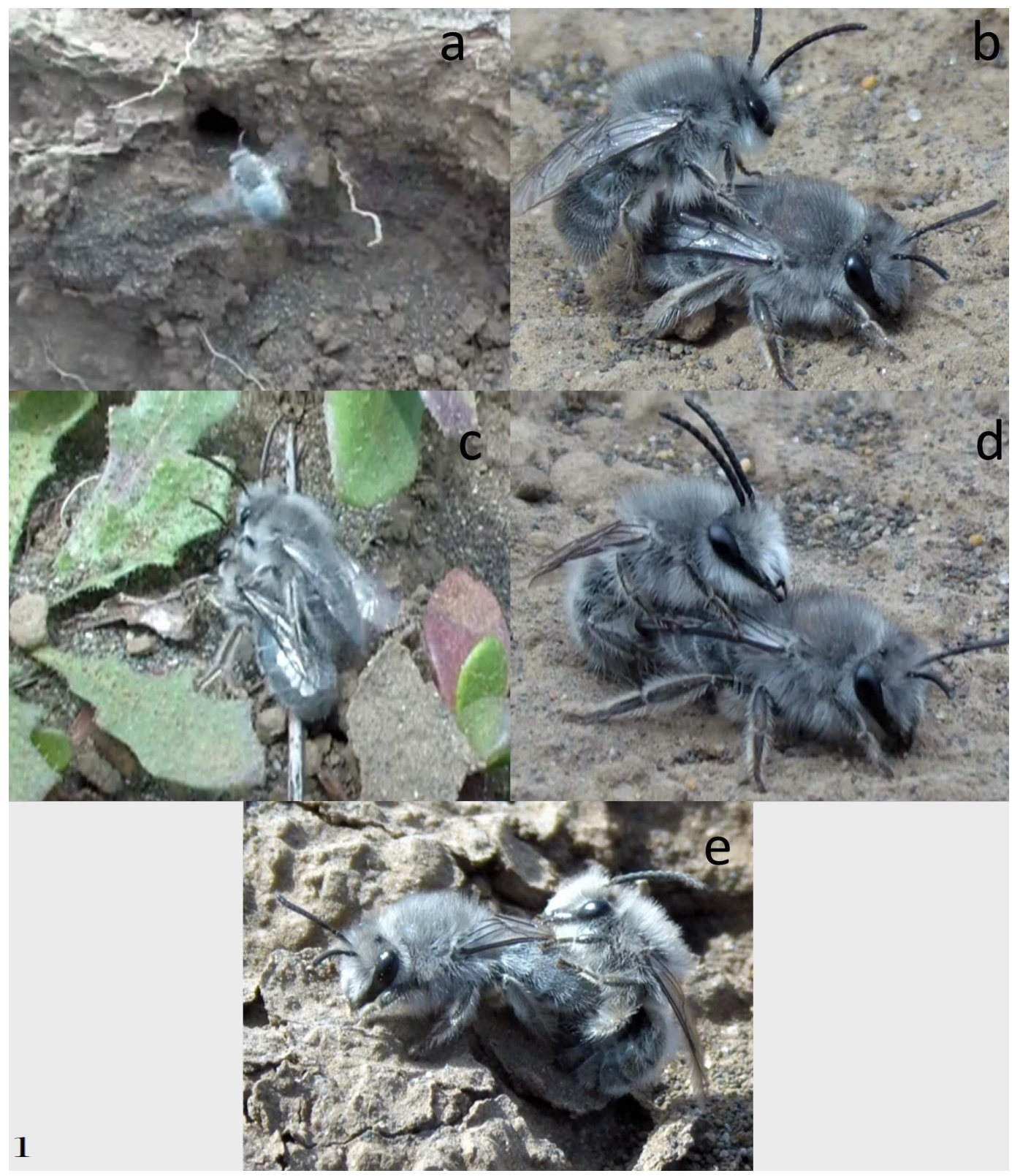

Figura 1. Comportamiento reproductivo de los machos de Colletes musculus antes y durante la cópula. a. Patrullaje, b. Contacto, c. Montaje, d. Fijación, e. Cópula. 
Tabla 1. Primera columna: lista de los comportamientos de apareamiento de Colletes musculus antes, durante y después de la cópula, segunda columna: las descripciones de esos comportamientos, tercera columna: resultados del índice de estereotipia en los comportamientos de apareamiento.

\begin{tabular}{|c|c|c|c|}
\hline \multirow[t]{2}{*}{ Conducta } & \multirow[t]{2}{*}{ Descripción } & \multicolumn{2}{|c|}{$\begin{array}{l}\text { Indice de } \\
\text { estereotipia }\end{array}$} \\
\hline & & Sli & SI, Parcial \\
\hline a. Patrullaje & $\begin{array}{l}\text { El } \widehat{\partial} \text { patrulla cerca de los nidos o entra ligeramente a los nidos } \\
\text { buscando } ㅇ \text { ․ }\end{array}$ & 1 & 0,17 \\
\hline b. Contacto & $\begin{array}{l}\text { El } \partial \widehat{~ s e ~ a p r o x i m a ~ a ~ l a ~}+\text { desde cualquier dirección mientras permanece } \\
\text { en el suelo. }\end{array}$ & 1 & 0,17 \\
\hline c. Montaje & 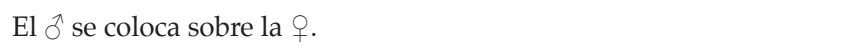 & 1 & 0,17 \\
\hline $\begin{array}{l}\text { d. Fijación de } \\
\text { la } q+\end{array}$ & $\begin{array}{l}\text { Las garras del primer par de patas del } \precsim \text { se fijan en la vena Costa del } \\
\text { ler par de alas de la }+.\end{array}$ & 1 & 0,17 \\
\hline e. Cópula & $\begin{array}{l}\text { El } \hat{~} \text { inserta la genitalia en el abdomen de la } \text { q. Durante la cópula, } \\
\text { el } \hat{\alpha} \text { con las alas plegadas sobre el abdomen realizá movimientos } \\
\text { ráapidos vibratorios, alternando con movimientos abdominales } \\
\text { anteroposteriores. }\end{array}$ & 1 & 0,17 \\
\hline f. Desmontaje & Separación de las genitalias de $\widehat{\partial}$ y + . & 1 & 0,17 \\
\hline \multirow[t]{2}{*}{ g. Partida } & El đ̧ abandona el área donde se realizó la cópula. & 0 & 0 \\
\hline & & \multicolumn{2}{|c|}{ SI, Global $=1,00$} \\
\hline
\end{tabular}

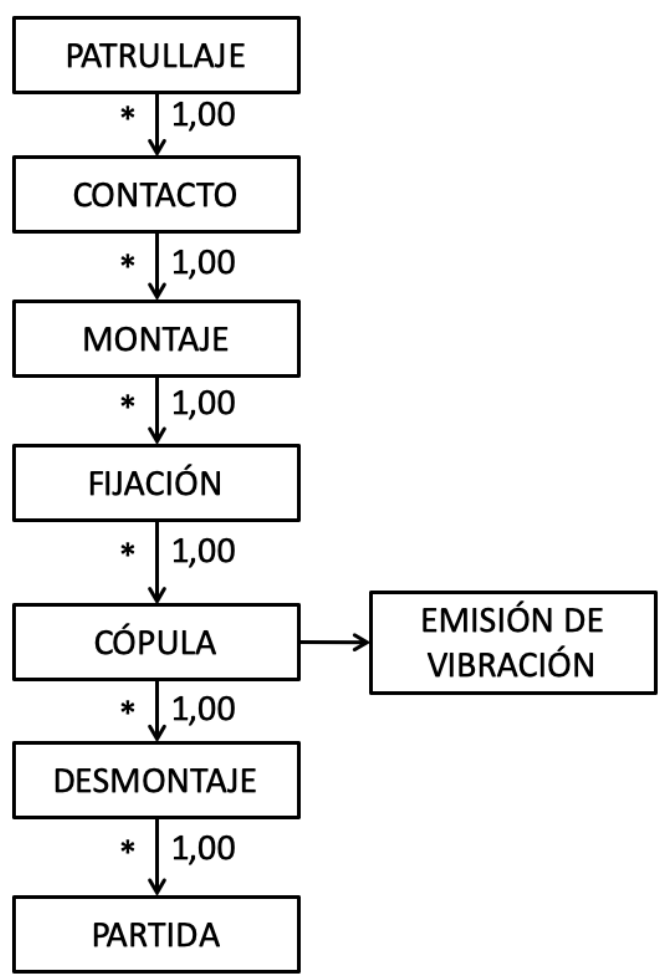

Figura 2. Etograma representando la conducta de cópula de los machos de Colletes musculus. Se indica la significación de las transiciones ${ }^{*}=\mathrm{p}<0,05$. 
Durante las cópulas, otros machos están frecuentemente intentando tomar a las hembras, en esos casos los machos en cópula sueltan a la hembra, elevan su cuerpo y se defienden con sus patas anteriores y medias, rechazando fuertemente a los competidores.

Los resultados muestran que los elementos durante la conducta reproductiva de los machos, las conductas previas, se exhiben con una probabilidad del $100 \%$ ya que son esenciales para el éxito de la cópula. Además, se presentan altamente estereotipados (SI = 100), no presentando variaciones en el comportamiento. El etograma resultante con estos comportamientos se presentan y resumen en Figs. 1-2 y Tabla 1.

C. musculus emite un sonido detectable durante la ocurrencia de la cópula en forma diferencial durante las distintas etapas de este proceso y se caracteriza por concentrar su energía de emisión entre los armónicos 14 y 35 que corresponden a las frecuencias promedio de 7,098 $\mathrm{Hz}$ y 14,117, respectivamente (Tabla 2) que pertenece a un rango de sonido muy agudo. Los sonidos representados en los sonogramas muestran la vibración de las alas en un período de 23 segundos (Fig. 3) y también las emisiones completas de los sonidos durante la copulación (Fig. 4). Las emisiones sonoras en C. musculus coinciden con la periodicidad de las emisiones expresadas en segundos, que se encuentra entre 2.34.8 s. El análisis estadístico de los parámetros de sonido se muestra en la Tabla 2.

Tabla 2. Muestra los valores estadísticos versus los parámetros de emisiones sonoras generadas por la vibración de las alas de los machos durante la cópula de Colletes musculus.

\begin{tabular}{|l|l|l|l|l|l|l|l|}
\hline Rarámetros & $\begin{array}{l}\text { Duración } \\
\text { del } \\
\text { sonido } \\
\text { (ms) }\end{array}$ & $\begin{array}{l}\text { Intervalo } \\
\text { entre } \\
\text { sonidos } \\
(\mathrm{ms})\end{array}$ & $\begin{array}{l}\text { Frequencia } \\
\text { del } \\
\text { fundamental } \\
(\mathrm{Hz})\end{array}$ & $\begin{array}{l}\text { Número } \\
\text { del } \\
\text { armónico } \\
\text { inicial }\end{array}$ & $\begin{array}{l}\text { Frecuencia } \\
\text { del } \\
\text { armónico } \\
\text { inicial } \\
(\mathrm{Hz})\end{array}$ & $\begin{array}{l}\text { Número } \\
\text { del } \\
\text { armónico } \\
\text { final }\end{array}$ & $\begin{array}{l}\text { Frecuencia } \\
\text { del } \\
\text { armónico } \\
\text { final (Hz) }\end{array}$ \\
\hline Min & 23,00 & 139,00 & 440,00 & 14,00 & $6.160,00$ & 30,00 & $13.200,00$ \\
\hline Max & 51,00 & 288,00 & 450,00 & 18,00 & $8.100,00$ & 35,00 & $15.750,00$ \\
\hline Promedio & 36,32 & 198,86 & 445,85 & 16,13 & $7.098,52$ & 31,37 & $14.117,85$ \\
\hline D/S & 7,36 & 39,61 & 2,98 & 1,05 & 460,68 & 1,38 & 622,72 \\
\hline
\end{tabular}

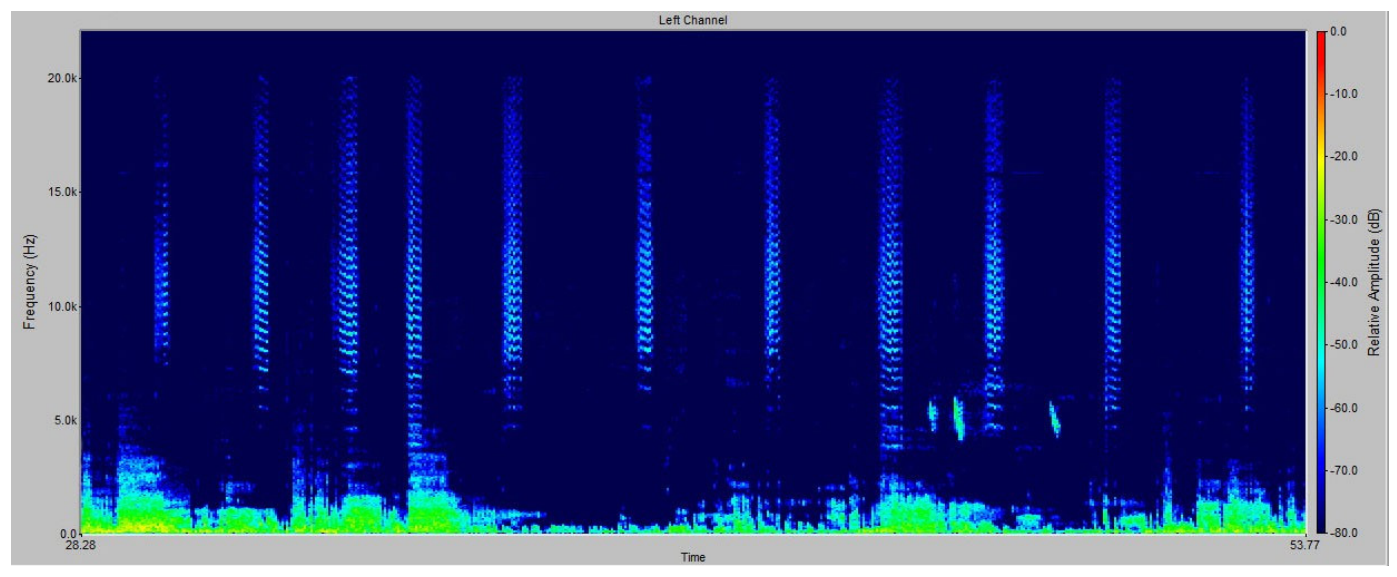

Figura 3. Sonograma de la secuencia de emisión de 23 segundos de sonido durante la cópula de un macho de Colletes musculus. 


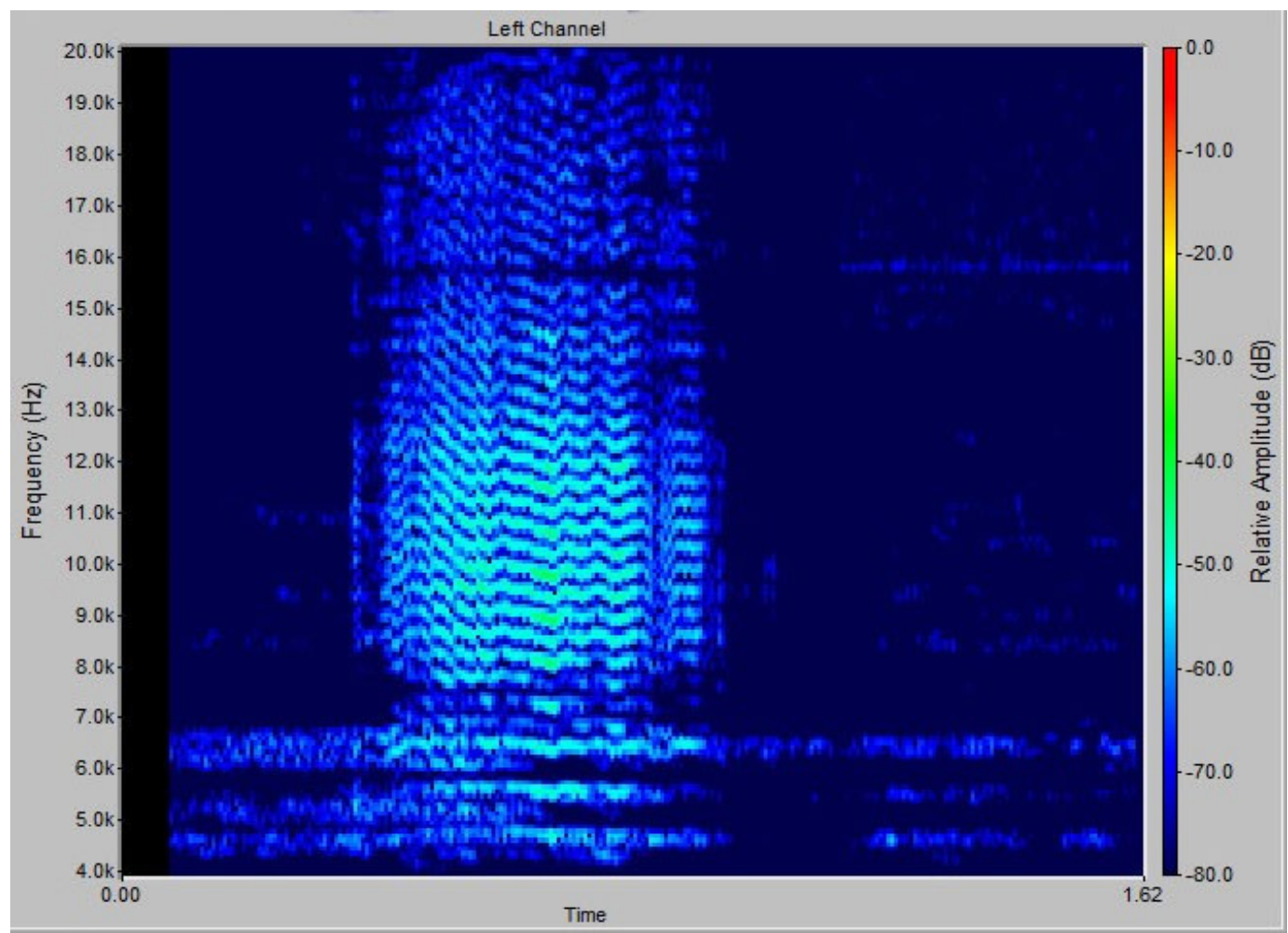

Figura 4. Sonograma de emisiones sonoras realizadas por Colletes musculus durante la cópula.

\section{Discusión}

Los machos de C. musculus presentan un tipo de estrategia reproductiva que implica ubicar a las hembras en las proximidades del sitio de nidificación, un comportamiento que aparece en la mayoría de las especies de abejas (Paxton 2015). La distribución en el espaciotiempo de las hembras receptivas y la historia de su vida reproductiva, en su conjunto, determinan la fuerza de la selección sexual en la especie (Paxton 2005).

Sobre esta especie se ha observado que los machos buscan a las hembras en momentos específicos del año y las horas del día (Chiappa et al. 2018). Esto está relacionado con el tipo de clima y hábitat donde viven las especies, lo que determina el tipo de sistema de apareamiento (Peackal y Schiestl 2004).

Respecto a ello, las condiciones de la Región de La Araucanía, donde se encuentra el área de nidificación de esta especie en particular, presenta un clima muy duro, con una alta tasa de precipitaciones (Di Castri y Hayek 1976; Luebert y Pliscoff 2006). De acuerdo con estas características ambientales, la temporada reproductiva de C. musculus es, adaptativamente, muy corta y con una breve protandria.

Los machos de esta especie, buscan activamente hembras dentro del área donde nidifican, mostrando un comportamiento del sistema de competencia de lucha no territorial (scramble competition system) (Paxton 2015), el sistema más frecuente en abejas (Chiappa 1996; Seidelmann 1999; Paxton 2005). La alta densidad de nidos y la aparición casi sincronizada de hembras (Chiappa et al. 2018) maximizan el éxito reproductivo de los machos que buscan hembras emergidas recientemente cerca de las áreas de nidificación (Thornhill y Alcock 1983). Como la densidad de los nidos es alta, la densidad de las hembras también es mayor; es decir, se ejerce presión sobre la selección para que el apareamiento tenga lugar en la mayor proximidad a los nidos. 
Además, C. musculus presenta dimorfismo sexual. Este patrón apoya la predicción con respecto al tamaño y el comportamiento en relación con la búsqueda de las hembras por los machos, debido al hecho de que hay poca ventaja selectiva para que los machos presenten mayor tamaño como en especies no territoriales que también presentan un sistema de competencia por lucha (scramble competition system, Alcock y Buchmann 1985). Ese no es el caso de las especies territoriales donde los machos son del mismo tamaño o más grandes que las hembras, como es el caso de C. cunicularius y otras especies territoriales de abejas (Larsson y Tengö 1989; Paxton y Tengö 1996; Seidelmann 1999).

Según las observaciones, claramente las hembras copulan solamente con algunos machos, esto plantea el supuesto que la hembra ya estaba fertilizada y que, como la mayoría de las abejas, solo reciben una cópula (Eickworth y Ginsberg 1980). Esto se corrobora cuando se pudo observar en dos ocasiones, que la hembra por no haber podido rechazar al macho, expulsó fuertemente el semen luego de realizada la cópula. Experiencias realizadas mostraron que las hembras que no reciben este despliegue del sonido, en abejas de la especie Centris pallida, retienen la receptividad y copulan nuevamente (Alcock y Bushman 1985).

Contextualizada la situación ecológica en la que se desarrolla la especie (Chiappa et al. 2018), es interesante haber categorizado los comportamientos que los machos realizan durante la cópula y representarlos en un etograma que resume las etapas del apareamiento, principalmente señalando el momento en que el macho emite sonidos y posibilitando la oportunidad de comparar los comportamientos de cópula con otras especies del género (Figs. 1-2).

La secuencia obtenida no ocurre por casualidad, la relación entre los comportamientos es total, unidireccional y similar a muchos otros insectos, porque C. musculus no muestra flexibilidad de comportamiento antes y durante el apareamiento. Esto se ha reflejado en el alto índice de estereotipia obtenido, y representa una gran ritualización sin mucho espacio para la ambigüedad con respecto a las señales observadas (Osorio y Cibrian 2000; Girling y Cardé 2006; Curkovic y Muñoz 2011; Mandujano et al. 2016).

En relación con la emisión de sonidos, existen especies de abejas, en las cuales los machos emiten sonidos sólo durante el contacto genital, producidas por vibraciones que pueden variar según la temperatura ambiental como en C. cunicularius (Larsen et al. 1986) y que disminuyen antes del final de la cópula. Los estudios realizados sobre las emisiones sonoras generadas durante o después de la cópula en el género Centris, mostraron cambios en la duración, la concentración de energía, en armónicos fundamentales y en los intervalos (Alcock y Bushmann 1985; Toro y Riveros 1998), probablemente debido al hecho que la emisión del sonido durante la cópula se produce al frotar los segmentos abdominales, no por los movimientos de las alas, como ocurre en esta especie de C. musculus.

Se han propuesto pocas interpretaciones con respecto a la función que estos sonidos emitidos por los machos podrían tener. En el caso de C. pallida, dado que su ocurrencia es al final de la cópula, se hipotetizó que servirían para marcar a las hembras para que no copularan con otros machos. Las vibraciones emitidas por los machos cambian la receptividad de las hembras, como se sugirió también para Colletes cunicularius en que el macho emite sonidos regularmente durante la cópula (Manning 1967; Alcock y Buchmann 1985; Larsen et al. 1986).

En el caso de C. musculus, el sonido emitido por el macho ocurre durante la cópula, lo que sugiere su intervención en el proceso de selección sexual, ya que podría reflejar la calidad sexual de los machos contra los competidores dentro de un sistema de apareamiento de competencia de lucha no territorial y también, como en el caso de las especies de Centris mencionadas anteriormente, para cambiar la capacidad de respuesta en las hembras. Para la abeja chilena C. mixta, el sonido ha sido interpretado como un esfuerzo reproductivo durante la eyaculación (Toro y Riveros 1998), que no refleja lo que sucede con C. musculus. 
En otros animales que usan este tipo de frecuencia en sus emisiones sonoras, las señales generalmente están relacionadas con conductas de alarma, conflicto o disociación (Collias 1960; Cocroft y Rodríguez 2005), por lo que este tipo de sonido en C. musculus podría interpretarse como una señal de advertencia para inducir a otros machos a desistir y para evitar intentos de cópula cuando la pareja está bloqueada genitalmente y, como resultado de esta vibración realizada por los machos durante la cópula, las hembras copulan solo una o muy pocas veces.

\section{Agradecimientos}

Agradecemos al Dr. Edoardo Tosti-Crose A. de la Universidad de la Frontera por su inestimable ayuda con las observaciones de campo.

\section{Literatura Citada}

Alcock, J. y Buchmann, S.L. (1985) The significance of post-insemination display by male Centris pallida (Hymenoptera: Anthophoridae). Zeitschrift für Tierpsychologie, 68: 231243.

Cade, W. (1985) Insect mating and courtship behaviour. Comprehensive Insect Physiology Biochemistry and Pharmacology, 9: 591-619.

Cane, J.H. (1987) Estimation of bee size using intertegular span (Apoidea). Journal of the Kansas Entomological Society, 60(1): 145-147.

Chiappa, E. (1996) Comportamiento reproductivo de machos de Sphex latreillei Lepeletier (Hymenoptera: Sphecidae). Revista Chilena de Entomología, 23: 19-27.

Chiappa, E., Araya, H., Mandujano, V. y Tosti-Croce, E. (2018) Descripción de los estados inmaduros de Colletes musculus Friese (Hymenoptera: Colletidae), con notas ecológicas y biológicas. Revista Chilena de Entomología, 44(2): 123-134.

Cocroft, R.B. y Rodríguez, L. (2005) The behavioral ecology of insect vibrational communication. BioScience, 55(4): 323-334.

Collias, N.E. (1960) An ecological and functional classification of animal sounds. pp. 368-391. En: Lanyon, W.E. y Tavolga, W.N. (Eds.) Animal Sounds and Communication. American Institute of Biological Sciences, Washington.

Conrad, T., Paxton, R.J., Barth, F.G., Francke, W. y Ayasse, M. (2010) Female choice in the red mason bee, Osmia rufa (L.) (Megachilidae). Journal of Experimental Biology, 213: 4065-4073.

Curkovic, T. y Muñoz, J. (2011) Caracterización del cortejo y cópula en Callisphyris apicornis: herramienta para definir la vialibilidad para desarrollar estrategias de manejo. Agrociencia, 45: 453-464.

Di Castri, F. y Hayek, E. (1976) Bioclimatología de Chile. Vicerrectoría Académica, Universidad de Chile, Santiago. 163 pp.

Eickworth, G.C. y Ginsberg, H.S. (1980) Foraging and mating in Apoidea. Annual Review of Entomology, 25: 421-446.

Fagen, R. y Young, D. (1978) Temporal patterns of behaviours: durations, intervals, latencies, and sequencies. pp. 79-114. En: Colgan P. (Ed.) Quantitative Ethology. Wiley, New York.

Girling, R. y Cardé, R. (2006) Analysis of the courtship behavior of the navel orangeworm, Amyelois transitella (Walker) (Lepidoptera: Pyralidae), with a commentary on methods for the analysis of sequences of behavioral transitions. Journal of Insect Behavior, 19: 497-520.

Hölldobler, B. y Wilson, E.O. (1990) The Ants. Springer, Berlin. 732 pp.

Hill, P.S. (2008) Vibrational communication in animals. Harvard University Press. 261 pp. 
Janvier, H. (1926) Recherches biologiques sur les Hyménoptères du Chili. Annales des Sciences Naturelles, 10(9): 113-349.

Larsen, O.N., Gleffe, G. y Tengö, J. (1986) Vibration and sound communication in solitary bees and wasps. Physiological Entomology, 11: 287-296.

Larsson, F.K. y Tengö, J. (1989) The effect of temperature and body size on the mating pattern of a gregariously nesting bee Colletes cunicularius (Hymenoptera: Colletidae). Ecological Entomology, 14: 279-286.

Leonhardt, S., Florian, D., Nehring, M.V. y Schmitt, T. (2016) Ecology and evolution of communication in social insects. Cell, 164: 1277-1287.

Luebert, F. y Pliscoff, P. (2006) Sinopsis bioclimótica y vegetacional de Chile. Universitaria SA, Santiago de Chile. 316 pp.

Mandujano, V., Flores-Prado, L. y Chiappa, E. (2016) Behavioural analysis and ethogram of mating in the wasp Sphex latreillei (Lepeletier) (Hymenoptera: Crabronidae). Neotropical Entomology, 45: 369. doi: https: / / doi.org/10.1007/s13744-016-0378-x

Manning, A. (1967) The control of sexual receptivity in female Drosophila. Animal Behaviour, 15: 239-250.

Markl, H. (1983) Vibrational communication. pp. 332-353. En: Huber, F. y Markl, H. (Eds.). Neuroethology and Behavioural Physiology: Roots and Growing Points. Springer, Berlin.

Montalva, J. y Ruz, L. (2010) Actualización de la lista sistemática de las abejas chilenas (Hymenoptera: Apoidea). Revista Chilena Entomología, 35: 15-52.

Osorio, R. y Cibrian, J. (2000) Conducta de cortejo del barrenador de la caña de azúcar Diatraea considerata Heinrich (Lepidoptera: Pyralidae). Agrociencia, 34: 619-626.

Paxton, R.J. y Tengö, J. (1996) Intranidal mating, emergence and sex ratio in a comunal bee Andrena jacobi Perkins 1921 (Hymenoptera: Andrenidae). Journal of Insect Behavior, 9: 421-440.

Paxton, R.J. (2005) Male mating behaviour and mating systems of bees: an overview. Apidologie, 36: 145-156.

Paxton, R.J. (2015) The bee-all and end-all. Nature, 521(7552): S57-S59.

Peackal, R. y Schiestl, F.P. (2004) A mark-recapture study of male Colletes cunicularius bees: implications for pollination by sexual deception. Behavioral Ecology and Sociobiology, 56: 579-584.

Peláez, F. y Veá, J. (1997) Etología: Bases biológicas de la conducta animal y humana. Ediciones Pirámide SA, Madrid. 395 pp.

Seidelmann, K. (1999) The race for females: the mating system of the red mason bee, Osmia rufa (Hymenoptera: Megachilidae). Journal of Insect Behavior, 12: 13-25.

Thornhill, R. (1979) Male and female sexual selection and the evolution of mating strategies in insects. pp. 81-121. En: Blum, M.S. y Blum, N.A. (Eds.). Sexual Selection and Reproductive Competition in Insects. Academic Press, New York.

Thornhill, R. y Alcock, J. (1983) The evolution of Insect Mating Systems. Harvard University Press, Cambridge. Massachusetts and London, England. 547 pp.

Toro, H. y Riveros, G. (1998) Comportamiento de cópula de Centris mixta tamarugalis (Hymenopera: Anthophoridae). Revista Chilena de Entomología, 25: 69-75.

Vander Meer, R., Breed, M., Winston, M. y Espelie, K. (1998) Pheromone Communication In Social Insects. CRC Press, Boca Raton. doi: https: / / doi.org/10.1201/9780429301575

Wisclo, W.T., Minckley, R.L. y Spangler, H.C. (1992) Pre-copulatory courtship behavior in a solitary bee, Nomia triangulifera Vachal (Hymenoptera: Halictidae). Apidologie, 23(5): 431-442. 